\title{
Thermal and gravitational chiral anomaly induced magneto-transport in Weyl semimetals
}

\author{
Kamal Das* and Amit Agarwal $\odot^{\dagger}$ \\ Department of Physics, Indian Institute of Technology Kanpur, Kanpur 208016, India
}

(Received 24 September 2019; published 28 January 2020)

\begin{abstract}
Quantum anomalies in Weyl semimetal (for either $\mathbf{E} \cdot \mathbf{B} \neq 0$ or $\nabla T \cdot \mathbf{B} \neq 0$ ) leads to chiral charge and energy pumping between the opposite chirality nodes. This results in chiral charge and energy imbalance between the Weyl nodes which manifests in several intriguing magneto-transport phenomena. Here, we investigate the role of electrical-, thermal-, and gravitational chiral anomaly on magneto-transport in Weyl semimetals. We predict the planar Ettinghausen and Righi-Leduc effect to be a distinct signature of these quantum anomalies. We also demonstrate a significant enhancement in the thermoelectric conductivity, Seebeck effect, Nernst effect, and thermal conductivity with increasing temperature. Interestingly, this anomaly induced transport violates the Wiedemann-Franz law and Mott relation.
\end{abstract}

DOI: 10.1103/PhysRevResearch.2.013088

\section{INTRODUCTION}

Massless chiral fluids in the presence of a magnetic field exhibit chiral anomalies, which manifest as the nonconservation of chiral charge and energy densities [1-6]. Weyl semimetals (WSMs) hosting a pair of Weyl nodes of opposite chirality, which act as Berry curvature monopoles [7-12], offer an ideal platform to explore these. The nonconservation of chiral charge in the presence of electric field $(\mathbf{E} \cdot \mathbf{B} \neq 0)$ is known as electrical chiral anomaly (ECA) [1,3,13]. This leads to very interesting magneto-electric transport phenomena $[3,4,14-23]$ in WSMs. The nonconservation of chiral energy in the presence of temperature gradient $(\nabla T \cdot \mathbf{B} \neq 0)$ is a manifestation of the gravitational chiral anomaly (GCA) $[2,4,5]$. This leads to interesting signatures in magnetothermal transport in WSMs [5,24-33]. Here, we demonstrate another kind of chiral anomaly associated with chiral charge pumping in the presence of $\nabla T \cdot \mathbf{B} \neq 0$, the thermal chiral anomaly (TCA).

The origin of these chiral anomalies can be traced to the magnetic field induced equilibrium chiral charge and energy current $\left(\mathbf{j}_{e \text {, eq }}^{s}\right.$ and $\mathbf{j}_{\mathcal{E} \text {, eq }}^{s}$, respectively, with $s= \pm 1$ being the chirality). These are

$$
\begin{gathered}
\mathbf{j}_{e, \text { eq }}^{s}=-e\left(\mu \mathcal{C}_{0}^{s} \mathbf{B}+T \mathcal{C}_{1}^{s} \mathbf{B}\right), \\
\mathbf{j}_{\mathcal{E}, \text { eq }}^{s}=\mu^{2} \frac{\mathcal{C}_{0}^{s}}{2} \mathbf{B}+\mu T \mathcal{C}_{1}^{s} \mathbf{B}+T^{2} \mathcal{C}_{2}^{s} \mathbf{B} .
\end{gathered}
$$

Here, $\mathcal{C}_{n}^{s}$ are the coefficients of different chiral anomalies defined later. Equation (1) generalizes the chiral magnetic effect in WSMs to include finite temperature. In Eq. (1),

\footnotetext{
*kamaldas@iitk.ac.in

†amitag@iitk.ac.in
}

Published by the American Physical Society under the terms of the Creative Commons Attribution 4.0 International license. Further distribution of this work must maintain attribution to the author(s) and the published article's title, journal citation, and DOI.
$\mathcal{C}_{0}^{s}$ is the ECA coefficient, while $\mathcal{C}_{1}^{s}$ determines the charge pumping in the presence of a finite $\nabla T$. We will refer to it as the coefficient of TCA. In Eq. (2) the first two terms simply denote the energy carried by chiral charge current, while the third term $\propto \mathcal{C}_{2}^{s}$ captures the thermal component and it is known to be analogous to the GCA [2,4,5]. In relativistic chiral fluids, the chiral gravitational anomaly depends on the Riemann tensor and should in principle vanish in a flat space-time. However, the coupling constant that appears in the chiral gravitational anomaly in a relativistic scenario is identical to that appearing in the thermal transport of WSMs $\left[\mathcal{C}_{2}\right.$ in Eq. (2)]. Thus, the thermal transport in WSMs is an analog of the relativistic chiral (also called axial) gravitational anomaly even in flat space-time $[2,4,5]$.

In a nonequilibrium scenario, this chiral charge pumping is stabilized by internode scattering and results in chiral charge (different $\mu^{s}$ ) and energy imbalance (different $T^{s}$ ) in the two Weyl nodes (see Fig. 1). This charge and energy imbalance in the Weyl nodes gives rise to several interesting effects in magneto-transport experiments. In this paper, we present a unified framework for these three chiral anomalies in the Boltzmann transport formalism.

We explicitly calculate all the magneto-transport coefficients, and predict their magnetic field dependence, angular dependence (between $\mathbf{B}$ and $\mathbf{E}$ or $\nabla T$ ), and temperature scaling. In addition to the planar Hall and planar Nernst effect, we predict planar Ettinghausen and Righi-Leduc effects to be a manifestation of these anomalies. Remarkably, we find significant enhancement in the magnetic field induced thermoelectric conductivity, Seebeck and Nernst effects, and thermal conductivity with increasing temperature. We also demonstrate that the chiral anomaly induced transport coefficients violate the Wiedemann-Franz law, as well as the Mott relation in WSMs.

\section{CHIRAL CHEMICAL POTENTIAL AND TEMPERATURE}

The dynamics of charge carriers in a Weyl cone of a given chirality is described by the following equations for the 

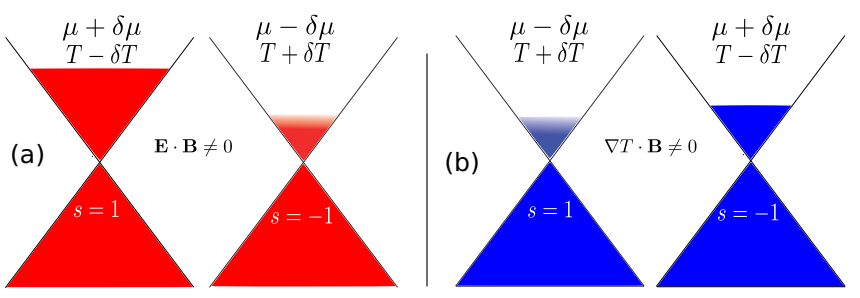

FIG. 1. Schematic of the chiral chemical potential $(\delta \mu)$ and chiral temperature $(\delta T)$ imbalance in WSMs for (a) $\mathbf{E} \cdot \mathbf{B} \neq 0$ and (b) $\nabla T \cdot \mathbf{B} \neq 0$. Both of these lead to quantum chiral anomalies, which pump chiral charge and energy from one Weyl node to the other.

carriers position (r) and momentum $(\mathbf{k})[34,35]$,

$$
\begin{aligned}
\dot{\mathbf{r}}^{s} & =\Delta^{s}\left[\tilde{\mathbf{v}}^{s}+\frac{e}{\hbar} \mathbf{E} \times \boldsymbol{\Omega}^{s}+\frac{e}{\hbar}\left(\tilde{\mathbf{v}}^{s} \cdot \boldsymbol{\Omega}^{s}\right) \mathbf{B}\right], \\
\hbar \dot{\mathbf{k}}^{s} & =\Delta^{s}\left[-e \mathbf{E}-e \tilde{\mathbf{v}}^{s} \times \mathbf{B}-\frac{e^{2}}{\hbar}(\mathbf{E} \cdot \mathbf{B}) \boldsymbol{\Omega}^{s}\right] .
\end{aligned}
$$

Here, " $-e$ " is the electronic charge, $\boldsymbol{\Omega}^{s}$ is the Berry curvature, and $\Delta^{s} \equiv 1 /\left(1+\frac{e}{\hbar} \boldsymbol{\Omega}^{s} \cdot \mathbf{B}\right)$ is the phase-space factor [36]. In the presence of magnetic field, the energy dispersion of the carriers is modified to include the orbital magnetization, $\tilde{\epsilon}^{s}=$ $\epsilon^{s}-\mathbf{m}^{s} \cdot \mathbf{B}$. The resultant band velocity is $\tilde{\mathbf{v}}^{s}=\frac{1}{\hbar} \frac{\partial \tilde{\epsilon}^{s}}{\partial \mathbf{k}}$ [37]. In Eq. (3), the third term $\left(\tilde{\mathbf{v}}^{s} \cdot \boldsymbol{\Omega}^{s}\right) \mathbf{B}$ is the chiral magnetic velocity $[13,14]$ which gives rise to the equilibrium chiral charge and energy currents in Eqs. (1) and (2). It is the foundation for all three chiral anomalies discussed in this paper.

In the presence of external perturbations, the nonequilibrium distribution function $\left(g_{\mathbf{r}, \mathbf{k}}\right)$ for each node is given by [38],

$$
\frac{\partial g_{\mathbf{r}, \mathbf{k}}^{s}}{\partial t}+\dot{\mathbf{r}}^{s} \cdot \nabla_{\mathbf{r}} g_{\mathbf{r}, \mathbf{k}}^{s}+\dot{\mathbf{k}}^{s} \cdot \nabla_{\mathbf{k}} g_{\mathbf{r}, \mathbf{k}}^{s}=I_{\mathrm{coll}}\left\{g_{\mathbf{r}, \mathbf{k}}^{s}\right\} .
$$

To reach a steady state in the presence of chiral charge and energy pumping between the two Weyl nodes, the collision integral $\left(I_{\text {coll }}\right)$ should include both the intranode $\left(\tau_{0}\right)$ as well as the internode $\left(\tau_{v}\right)$ scattering time scales. Furthermore, owing to the chiral charge and energy pumping, each of the Weyl nodes is assumed to acquire a local equilibrium (LE) chemical potential $\mu^{s} \equiv \mu+\delta \mu^{s}[15,39]$ and temperature $T^{s}=$ $T+\delta T^{s}$. Within this approximation the steady-state collision integral is given by

$$
I_{\text {coll }}^{s}=-\frac{g_{\mathbf{r}, \mathbf{k}}^{s}-f\left(\tilde{\epsilon}^{s}, \mu^{s}, T^{s}\right)}{\tau_{0}}-\frac{g_{\mathbf{r}, \mathbf{k}}^{s}-f\left(\tilde{\epsilon}^{s}, \mu^{\bar{s}}, T^{\bar{s}}\right)}{\tau_{v}} .
$$

Here, $f\left(\tilde{\epsilon}^{s}, \mu^{s}, T^{s}\right)$ is the Fermi function with energy $\tilde{\epsilon}^{s}$, chemical potential $\mu^{s}$, and temperature $T^{s}$. The first term in Eq. (6) reflects the relaxation of $g_{\mathbf{r}, \mathbf{k}}^{s}$ to the LE of the same node via intranode scattering while the second term specifies its relaxation to the LE of the other node by internode scattering.

Substituting this collision integral in Eq. (5), and integrating over all momentum modes, we obtain the following equation for the particle number $\left(\mathcal{N}^{s}\right)$ dynamics:

$$
\frac{\partial \mathcal{N}^{s}}{\partial t}+\mathcal{C}_{0}^{s} e \mathbf{E} \cdot \mathbf{B}+\mathcal{C}_{1}^{s} \nabla T \cdot \mathbf{B}=\frac{\mathcal{N}^{s}-\mathcal{N}^{\bar{s}}}{\tau_{v}}
$$

This generalizes the semiclassical ECA $\left(\propto \mathcal{C}_{0}^{s}\right)$ [3] to include the temperature gradient induced chiral charge pumping, or TCA $\left(\propto \mathcal{C}_{1}^{s}\right)$. Similarly we calculate the energy dynamics to be

$$
\begin{aligned}
& \frac{\partial \mathcal{E}^{s}}{\partial t}+\left(\mu \mathcal{C}_{0}^{s}+T \mathcal{C}_{1}^{s}\right) e \mathbf{E} \cdot \mathbf{B}+\left(\mu \mathcal{C}_{1}^{s}+2 T \mathcal{C}_{2}^{s}\right) \nabla T \cdot \mathbf{B} \\
& =\frac{\mathcal{E}^{s}-\mathcal{E}^{\bar{s}}}{\tau_{v}} .
\end{aligned}
$$

Here, the $\mu \mathcal{C}_{0}^{s}$ and the $\mu \mathcal{C}_{1}^{s}$ terms represent the energy carried by the chiral charge transfer. The $2 T \mathcal{C}_{2}^{s}$ term highlights the energy pumped by the $\nabla T \cdot \mathbf{B} \neq 0$ term and it is analogous to GCA.

Working in the linear-response regime in $\mathbf{E}$ and $\nabla T$, we find that the imbalance of the chiral carriers and temperatures is small, i.e., $\delta \mu^{s}<\mu$, and $\delta T^{s}<T$. Solving for $\delta \mu^{s}$ and $\delta T^{s}$ (see Appendix B for more details) in this regime, we obtain

$$
\left(\begin{array}{c}
\delta \mu^{s} \\
\delta T^{s} / T
\end{array}\right)=-\frac{\tau_{v}}{2}\left(\begin{array}{ll}
\mathcal{D}_{0}^{s} & \mathcal{D}_{1}^{s} \\
\mathcal{D}_{1}^{s} & \mathcal{D}_{2}^{s}
\end{array}\right)^{-1}\left(\begin{array}{cc}
\boldsymbol{\Lambda}_{0}^{s} & \boldsymbol{\Lambda}_{1}^{s} \\
\boldsymbol{\Lambda}_{1}^{s} & \boldsymbol{\Lambda}_{2}^{s}
\end{array}\right) \cdot\left(\begin{array}{c}
e \mathbf{E} \\
\nabla T / T
\end{array}\right) .
$$

Here, we have defined the generalized energy densities and the generalized energy velocities at the Fermi energy,

$$
\left(\begin{array}{c}
\mathcal{D}_{n}^{s} \\
\boldsymbol{\Lambda}_{n}^{s}
\end{array}\right)=\int \frac{d \mathbf{k}}{(2 \pi)^{3}}\left(\tilde{\epsilon}^{s}-\mu\right)^{n}\left(-\partial_{\tilde{\epsilon}^{s}} f\right)\left(\begin{array}{c}
1+e \boldsymbol{\Omega}^{s} \cdot \mathbf{B} / \hbar \\
\tilde{\mathbf{v}}^{s}+\frac{e}{\hbar}\left(\tilde{\mathbf{v}}^{s} \cdot \boldsymbol{\Omega}^{s}\right) \mathbf{B}
\end{array}\right),
$$

with $n=\{0,1,2\}$. Here, $\mathcal{D}_{0}^{s}$ defines the finite temperature density of states in the presence of Berry curvature. $\boldsymbol{\Lambda}_{0}^{s}$ and $\boldsymbol{\Lambda}_{1}^{s}$ are the total chiral magnetic velocity and the total chiral energy velocity at the Fermi level, respectively. It is evident from Eq. (9) that both the electric field and the temperature gradient contribute to generate the chiral chemical potential and chiral temperature imbalance in the WSMs. It turns out that in $\boldsymbol{\Lambda}_{n}^{s}$, only the contribution of the chiral magnetic velocity survives and we have $\boldsymbol{\Lambda}_{n}^{s}=\Lambda_{n}^{s} \mathbf{B}$, along with $\Lambda_{n}^{s} \propto s$. Note that in Eq. (9), the energy and temperature dependence of the scattering time can be included by replacing $\tau_{v} \rightarrow \tau_{v}(\mu, T)$ (see Appendixes B and D for more detail).

The anomaly coefficients are explicitly given by

$$
\left\{\mathcal{C}_{0}^{s}, \mathcal{C}_{1}^{s}, \mathcal{C}_{2}^{s}\right\}=\left\{\Lambda_{0}^{s}, \frac{\Lambda_{1}^{s}}{T}, \frac{\Lambda_{2}^{s}}{2 T^{2}}\right\}
$$

Clearly, in this semiclassical formalism, all these three anomalies arise from the presence of a finite Berry curvature and chiral magnetic velocity. However, while the chiral anomaly $\left(\propto \mathcal{C}_{0}^{s}\right)$ is well explored, the other two anomalies of a similar origin are relatively less explored [4,5]. Developing a common theoretical framework to explore all of them is one of the main highlights of this paper.

\section{ANOMALY INDUCED TRANSPORT COEFFICIENTS}

The anomaly induced contribution to the charge $\left(\mathbf{j}_{e}=\right.$ $\left.\sum_{s} \mathbf{j}_{e}^{s}\right)$ and heat $\left(\mathbf{j}_{Q}=\sum_{s} \mathbf{j}_{Q}^{s}\right)$ current can now calculated to

$$
\left(\begin{array}{c}
\mathbf{j}_{e}^{s} \\
\mathbf{j}_{Q}^{s}
\end{array}\right)=\frac{\mathbf{B} \tau_{v}}{2}\left(\begin{array}{cc}
e \Lambda_{0}^{s} & e \Lambda_{1}^{s} \\
-\Lambda_{1}^{s} & -\Lambda_{2}^{s}
\end{array}\right) \mathcal{D}_{s}^{-1}\left(\begin{array}{cc}
\Lambda_{0}^{s} & \Lambda_{1}^{s} \\
\Lambda_{1}^{s} & \Lambda_{2}^{s}
\end{array}\right)\left(\begin{array}{c}
e \mathbf{B} \cdot \mathbf{E} \\
\mathbf{B} \cdot \nabla T / T
\end{array}\right),
$$


where

$$
\mathcal{D}_{s}=\left(\begin{array}{ll}
\mathcal{D}_{0}^{s} & \mathcal{D}_{1}^{s} \\
\mathcal{D}_{1}^{s} & \mathcal{D}_{2}^{s}
\end{array}\right) .
$$

Now, as $\Lambda_{n}^{s} \propto C_{n}^{s}$, Eq. (12) implies that the charge and heat current in WSMs are associated with ECA, TCA, GCA, or mixed anomalies (a combination of two of these). Thus Eq. (12) captures the essence of the chiral anomalies induced magneto-transport, and is one of the main results of this work. See Appendix C for the expanded version of Eq. (12). Note that the internode scattering involves large momentum transfers (of the order of the separation of the Weyl nodes) as compared to the intranode scattering [40]. Due to this we have $\tau_{v} \gg \tau$, and thus we have ignored the intranode contribution in Eq. (12).

The transport coefficients can be obtained by comparing Eq. (12) to the phenomenological linear response, $j_{e, i}=$ $\sum_{j}\left[\sigma_{i j} E_{j}-\alpha_{i j} \nabla_{j} T\right]$ and $j_{Q, i}=\sum_{j}\left[\bar{\alpha}_{i j} E_{j}-\bar{\kappa}_{i j} \nabla_{j} T\right]$. Here, $\sigma, \alpha, \bar{\alpha}$, and $\bar{\kappa}$ denote the electrical, thermoelectric, electrothermal, and constant voltage thermal conductivity matrix, respectively. The thermopower matrix is defined as $S_{i j}=$ $\left[\sigma^{-1} \alpha\right]_{i j}$ and the open circuit thermal conductivity is $\kappa_{i j}=$ $\left[\bar{\kappa}-\bar{\alpha} \sigma^{-1} \alpha\right]_{i j}$. The diagonal components of $S$ are the Seebeck components, while the off-diagonal components are the Nernst coefficients. Clearly, the different transport coefficients are determined by either the ECA, TCA, GCA, or a combination of two of these (mixed anomalies) [5]. Explicit analytical expressions of each of the transport coefficient matrixes and their association with different anomalies is discussed in Appendix C.

We find that the anomaly induced transport coefficients in WSMs satisfy Onsager's reciprocity relations, $T \delta \alpha_{i j}(B)=$ $\delta \bar{\alpha}_{j i}(-B)$ in addition to $\delta \sigma_{i j}(B)=\delta \sigma_{j i}(-B)$ and $\delta \bar{\kappa}_{i j}(B)=$ $\delta \bar{\kappa}_{j i}(-B)$. This is guaranteed by the fact that the $\mathcal{D}_{n}^{s}$ is an even function of $B$. The Onsagar's reciprocity relations in WSMs have also been shown to be valid in the hydrodynamic transport limit [4]. However, in contrast to normal metals, the electronic transport coefficients of Eq. (12) violates the Wiedemann-Franz law $[L=\kappa /(T \sigma)=$ const $]$ as well as the Mott relation $\left[M=\alpha /\left(\left.T \partial_{\epsilon} \sigma\right|_{\mu}\right)=\right.$ const $]$.

This summarizes our formulation for exploring magnetotransport in WSMs induced by the chiral anomalies. This framework can now be combined with ab initio based Wannier models, or few orbital based tight-binding models for material specific predictions. As long as a material (or a specific model) has Weyl nodes with finite chiral charges, the coefficients $\mathcal{C}_{0}, \mathcal{C}_{1}$, and $\mathcal{C}_{2}$ are finite, and it will have the signatures of these quantum chiral anomalies. More specifically, any WSM or a multi-WSM will show the predicted anomaly induced transport phenomena. Below, we present results for a lowenergy model of a WSM with a single pair of Weyl nodes.

\section{LOW-ENERGY MODEL OF WSM}

The low-energy Hamiltonian for a Weyl node is

$$
\mathcal{H}^{s}=s \hbar v_{F} \boldsymbol{\sigma} \cdot \mathbf{k},
$$

where $v_{F}$ is the Fermi velocity and $\sigma=\left\{\sigma_{1}, \sigma_{2}, \sigma_{3}\right\}$ is the set of Pauli spin matrices. The Berry curvature and the orbital magnetic moment for the conduction band of the Hamiltonian in Eq. (13) are $\boldsymbol{\Omega}^{s}=-s \mathbf{k} /\left(2|\mathbf{k}|^{3}\right)$ and $\mathbf{m}^{s}=-\operatorname{sev}_{F} \mathbf{k} /\left(2|\mathbf{k}|^{2}\right)$, respectively.

For this model Hamiltonian, it is straightforward to calculate the quantities defined in Eq. (10). The generalized energy velocities can be calculated to be

$$
\left\{\Lambda_{0}^{s}, \Lambda_{1}^{s}, \Lambda_{2}^{s}\right\}=-s \frac{e}{4 \pi^{2} \hbar^{2}}\left\{\mathcal{F}_{0}, \frac{1}{\beta} \mathcal{F}_{1}, \frac{1}{\beta^{2}} \mathcal{F}_{2}\right\} .
$$

Here, $\mathcal{F}_{i}$ 's are functions of $x=\beta \mu$ and we have defined $\mathcal{F}_{0}(x) \equiv 1 /\left(1+e^{-x}\right), \quad \mathcal{F}_{1}(x) \equiv x /\left(1+e^{x}\right)+\ln \left[1+e^{-x}\right]$, and $\mathcal{F}_{2}(x) \equiv \pi^{2} / 3-x\left(\frac{x}{1+e^{x}}+2 \ln \left[1+e^{-x}\right]\right)+2 \operatorname{Li}_{2}\left(-e^{-x}\right)$, with $\mathrm{Li}$ denoting the polylog function (see Appendix A for their temperature dependence). Similarly we calculate the generalized energy density of states in the limit $\mu>k_{B} T$, where Sommerfeld expansion is valid, as

$$
\left\{\mathcal{D}_{0}^{s}, \mathcal{D}_{1}^{s}, \mathcal{D}_{2}^{s}\right\} \approx \frac{\mu^{2}}{2 \pi^{2}} \frac{1}{\hbar^{3} v_{F}^{3}}\left\{\mathcal{F}_{0}, \frac{2}{\beta^{2} \mu} \mathcal{F}_{2}, \frac{1}{\beta^{2}} \mathcal{F}_{2}\right\} .
$$

Note that in calculating the generalized energy densities, we have neglected the magnetic field correction which is very small. In the limiting case of $\beta \mu \rightarrow \infty$, we have $\left\{\mathcal{F}_{0}, \mathcal{F}_{1}, \mathcal{F}_{2}\right\} \rightarrow\left\{1,0, \pi^{2} / 3\right\}$. In this limit, $\mathcal{C}_{1}^{s} \rightarrow 0$ implying that there is no TCA.

Using Eqs. (14) and (15) in Eq. (9), we evaluate $\delta \mu^{s}$ and $\delta T^{s}$ in the $\beta \mu \rightarrow \infty$ limit to be

$$
\left(\begin{array}{c}
\delta \mu^{s} \\
\delta T^{s} / T
\end{array}\right)=s \frac{\tau_{v} \hbar^{2} v_{F}^{3}}{\mu^{2}} \frac{1}{2 \hbar}\left(\begin{array}{cc}
\frac{e^{2}}{2} & -\frac{e k_{B}}{\beta \mu} \frac{\pi^{2}}{3} \\
-\frac{e^{2}}{\mu} & \frac{e}{T}
\end{array}\right)\left(\begin{array}{c}
\mathbf{B} \cdot \mathbf{E} \\
\mathbf{B} \cdot \nabla T
\end{array}\right) .
$$

For finite $\beta \mu$, their variation in the $\mu-T$ plane (for $s=1$ node) is shown in Fig. 2, and analytical results are presented in Appendix C. The $\mathbf{B} \cdot \mathbf{E}$ and $\mathbf{B} \cdot \nabla T$ terms compete with each other to change both $\delta \mu^{s}$ as well as $\delta T^{s}$.

The chiral anomaly induced transport coefficients $(\sigma, \alpha, \bar{\alpha}$, and $\bar{\kappa})$ can now be obtained from Eq. (12). In the $\beta \mu \rightarrow \infty$ limit, these are given by

$$
\left(\begin{array}{cc}
\sigma_{i j} & \alpha_{i j} \\
\bar{\alpha}_{i j} & \bar{\kappa}_{i j}
\end{array}\right)=\frac{\tau_{v}}{2} \frac{e^{2} v_{F}^{3}}{8 \pi^{2} \hbar} \frac{B^{2}}{\mu^{2}}\left(\begin{array}{cc}
e^{2} & \frac{2 \pi^{2}}{3} \frac{e k_{B}}{\beta \mu} \\
\frac{2 \pi^{2}}{3} \frac{e k_{B} T}{\beta \mu} & \frac{\pi^{2}}{3} k_{B}^{2} T
\end{array}\right) \mathcal{L}_{i j}(\theta, \phi) .
$$

Here, $(\theta, \phi)$ denotes the polar and azimuthal angle of the magnetic field. Note that in Eq. (17), $\alpha$ and $\bar{\kappa}$ are $\propto T$ while $\bar{\alpha} \propto T^{2}$. The angular dependence of all transport coefficients is given by the matrix

$$
\begin{aligned}
& \mathcal{L}(\theta, \phi) \\
& \quad=\left(\begin{array}{ccc}
\sin ^{2} \theta \cos ^{2} \phi & \frac{1}{2} \sin ^{2} \theta \sin 2 \phi & \frac{1}{2} \sin 2 \theta \cos \phi \\
\frac{1}{2} \sin ^{2} \theta \sin 2 \phi & \sin ^{2} \theta \sin ^{2} \phi & \frac{1}{2} \sin 2 \theta \sin \phi \\
\frac{1}{2} \sin 2 \theta \cos \phi & \frac{1}{2} \sin 2 \theta \sin \phi & \cos ^{2} \theta
\end{array}\right) .
\end{aligned}
$$

Coplanar $\mathbf{E}$ and $\mathbf{B}$ give rise to the planer Hall effect $\left(\sigma_{x y}\right)$ along with the planar Ettingshausen effect $\left(\bar{S}_{x y}=T S_{x y}\right)$. Coplanar $\nabla T$ and $\mathbf{B}$ result in the planar Nernst effect $\left(S_{x y}\right)$ and the planar Righi-Leduc effect $\left(\kappa_{x y}\right)$. We find that the angular dependence of $\kappa_{x y}$ and $S_{x y}$ is identical $(\propto \sin 2 \phi)$, in contrast to $\rho_{x y}$ which is $\propto-\sin 2 \phi$ as shown in Fig. 3(a).

To explore the impact of anomalies on longitudinal magneto-resistance (MR), we define generic 


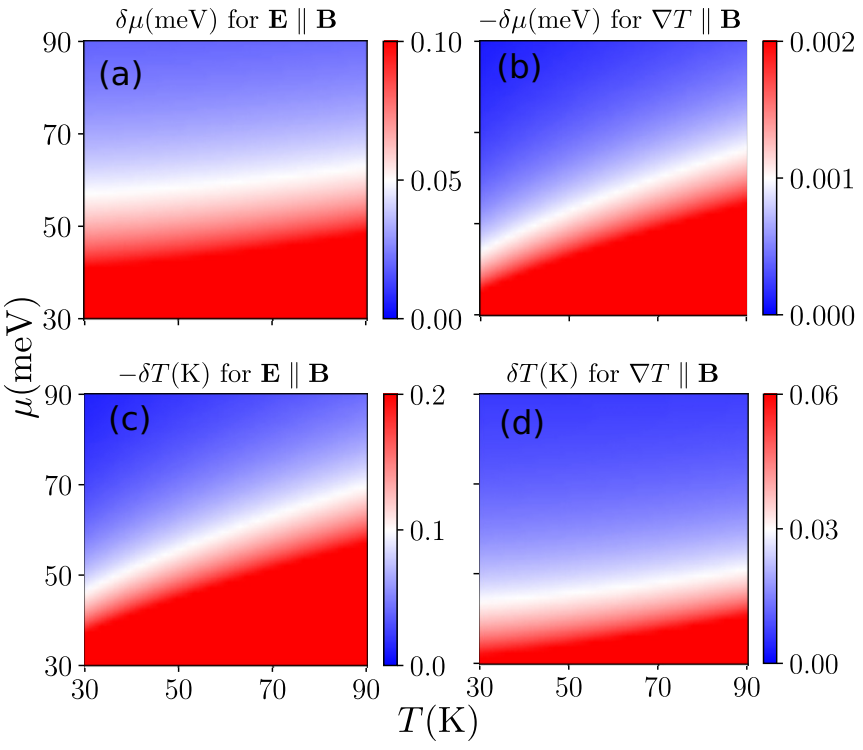

FIG. 2. The anomaly induced chiral chemical potential $\delta \mu^{s}$ and chiral temperature $\delta T^{s}$ for the $s=1$ node, in the $\mu-T$ plane. The $\delta \mu^{s}$ induced by (a) $\mathbf{E} \| \mathbf{B}$ and (b) $\nabla T \| \mathbf{B}$ have opposite signs. The $\delta T^{s}$ induced by (c) $\mathbf{E} \| \mathbf{B}$ and (d) $\nabla T \| \mathbf{B}$ also have opposite signs. In all cases, the impact of the $\mathbf{E} \| \mathbf{B}$ term is larger. Furthermore, while the $\mathbf{E} \cdot \mathbf{B} \neq 0$ term increases the chiral $\delta \mu$ of the positive chirality node, it reduces its chiral temperature $\delta T$. Here we have chosen $v_{F}=$ $2 \times 10^{5} \mathrm{~m} / \mathrm{s}, \tau_{v}=10^{-9} \mathrm{~s}, B=6 \mathrm{~T}$, sample length $l=50 \mu \mathrm{m},|\mathbf{E}|=$ $1 \mathrm{mV} / l$, and $|\nabla T|=350 \mathrm{mK} / l[5]$.

$\mathrm{MR}_{\gamma} \equiv \gamma(B) / \gamma(0)-1$, where $\gamma$ denotes the transport coefficients: $\sigma, \alpha, \bar{\alpha}, \kappa$, or $S$. To evaluate the MR, we need to add the Drude components to the respective transport coefficients. Evaluating the Drude components for each Weyl node [41], we have $\left\{\sigma_{0}^{s}, \alpha_{0}^{s}, \bar{\kappa}_{0}^{s}\right\}=\frac{\mu^{2} \tau_{0}}{6 \pi^{2} \hbar^{3} v_{F}}\left\{e^{2} \mathcal{F}_{0},-2 \frac{e k_{B}}{\beta \mu} \mathcal{F}_{2}, \frac{k_{B}}{\beta} \mathcal{F}_{2}\right\}$. In the $\beta \mu \rightarrow \infty$ limit, to all orders in $B$ we obtain the longitudinal $\mathrm{MR},\left\{\mathrm{MR}_{\rho}, \mathrm{MR}_{S}\right\}=-\frac{3 \tau_{v} \zeta^{2}}{2 \tau_{0}+3 \tau_{v} \zeta^{2}}\{1,2\}$. Here $\zeta=e \hbar v_{F}^{2} B /\left(2 \mu^{2}\right)$. Both of these show negative MR which is quadratic for small $B$ and saturates with increasing $B$ values. This behavior, along with $\mathrm{MR}_{\rho} / \mathrm{MR}_{S}=1 / 2$, persists even for finite $\beta \mu$ values, as shown in Fig. 3(b) for an electronically doped system $(\mu>0)$. Negative saturating $\mathrm{MR}_{\rho}$ in WSMs has been reported in several experiments [16-18]. Negative $\mathrm{MR}_{S}$ has also been reported in recent experiments on GdPtBi [26] and $\mathrm{Cd}_{3} \mathrm{As}_{2}$ [27].

Now, we focus on $\alpha$ and $\kappa$. In the $\beta \mu \rightarrow 0$ limit, we obtain $\left\{\mathrm{MR}_{\alpha}, \mathrm{MR}_{\bar{\kappa}}\right\}=\frac{3 \tau_{v} \zeta^{2}}{2 \tau_{0}}\{-1,1\}$. In contrast to $\mathrm{MR}_{\rho} / \mathrm{MR}_{S}$ and consistent with calculations of Ref. [28], both of these show a nonsaturating behavior with $\mathrm{MR}_{\alpha}$ being negative, and $\mathrm{MR}_{\bar{\kappa}}$ being positive. This trend persists even for finite $\beta \mu$ as shown in Figs. 3(c) and 3(d). The observation of semiclassical negative $\mathrm{MR}_{\alpha}$ (for $\mu>0$ ) has also been reported in the Weyl semimetal NbP [5] as well in GdPtBi [26]. A positive $\mathrm{MR}_{\kappa}$ has been recently reported in Ref. [42]. We emphasize that the relatively larger MR in $\alpha$ and $\kappa$ as compared to $\mathrm{MR}_{\rho}$ has its origin primarily in the $\mathcal{C}_{2}^{s}$ term or the GCA.

The temperature dependence of the diagonal and offdiagonal transport coefficients is shown in Figs. 4(a) and 4(b). While the Drude-Seebeck coefficient is negative for $\mu>0$, the quantum anomalies reverse its sign, making it positive.
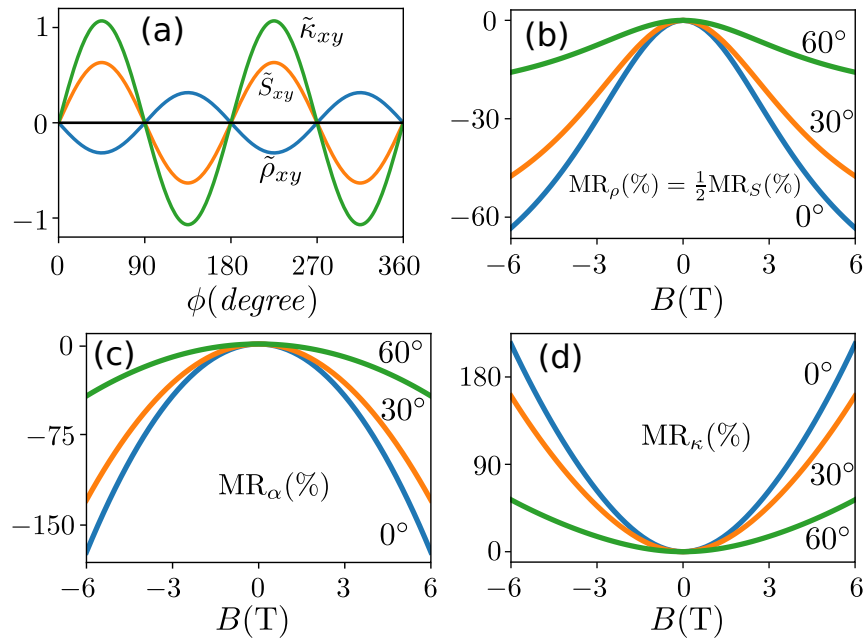

FIG. 3. Impact of the chiral anomalies induced magnetotransport coefficients in WSMs. (a) The planar Hall (blue), planar Nernst (orange), and the planar Righi-Leduc (green) effect (normalized by their longitudinal Drude counterpart) are shown as function of the angle $\phi$ between $\mathbf{B}$ and $\mathbf{E}$ or $\nabla T$. (b) The saturating negative magneto-resistance $\left(\mathrm{MR}_{\rho}\right)$ and negative magneto-Seebeck effect $\left(\mathrm{MR}_{S}\right)$. (c) The nonsaturating negative magneto thermoelectric conductivity $\left(\mathrm{MR}_{\alpha}\right)$ and (d) the positive magnetothermal conductivity. Here we have chosen $T=40 \mathrm{~K}, \mu=0.05 \mathrm{eV}$, and $\tau_{0}=10^{-12} \mathrm{~s}$, with all the other parameters being identical to those of Fig. 2.

This sign reversal is a distinct signature of quantum anomalies in chiral fluids (see Fig. 7). The Drude thermal conductivity is positive, and the chiral anomalies enhance it significantly [by
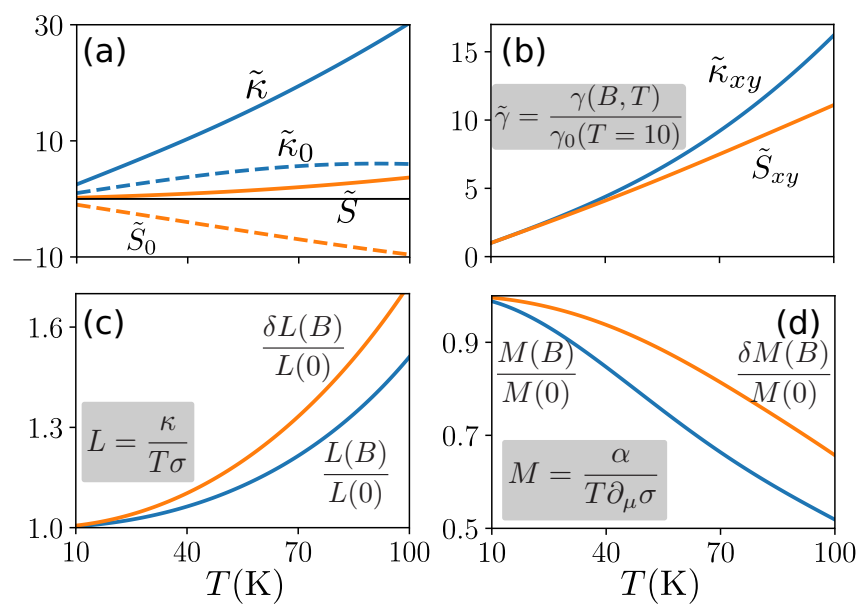

FIG. 4. (a) The $T$ dependence of Seebeck coefficient and thermal conductivity (scaled by their Drude values at $10 \mathrm{~K}$ ). The Drude $\kappa_{0}$ (blue dashed line) is positive and GCA enhances it significantly (blue solid line). The Drude $S_{0}$ is negative (orange dashed line) for $\mu>0$, but it flips sign in presence of magnetic field (orange solid line). (b) The GCA induced enhancement with temperature is also evident in the planar $\kappa_{x y}$ and $S_{x y}$ (scaled by their values at $10 \mathrm{~K}$ ) when the angle between $\mathbf{E}$ or $\nabla T$ and $\mathbf{B}$ is $45^{\circ}$. (c) The quantum anomalies induced violation of the Wiedemann-Franz law and (d) the Mott relation. Here, all the parameters are identical to those used for Figs. 2 and 3. 

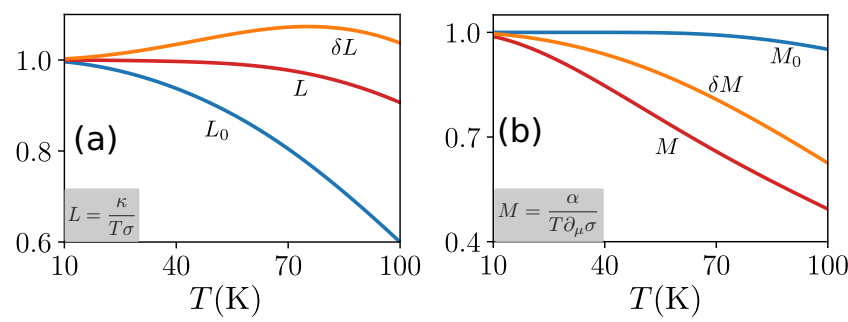

FIG. 5. The magnetic field induced violation of WiedemannFranz law and Mott relation (deviation from the $L_{0}$ and $M_{0}$ curves). Panel (a) shows the deviation of $L_{0}=\frac{\kappa_{0}}{T \sigma_{0}}$ (blue line), $L=\frac{\kappa}{T \sigma}$ (red line) and $\delta L_{0}=\frac{\kappa-\kappa_{0}}{T\left(\sigma-\sigma_{0}\right)}$ (orange line) from the conventional Lorentz number $\mathcal{L}_{0}$ which we have taken to be 1 . Panel (b) shows the variation of the Mott number from its conventional value $\mathcal{M}_{0}$ which is again taken to be 1 . Here, we have defined $M_{0}=\frac{\alpha_{0}}{T \sigma_{0}^{\prime}}$ (blue line), $M=\frac{\alpha}{T \sigma^{\prime}}$ (red line), and $\delta M=\frac{\delta \alpha}{T \delta \sigma^{\prime}}$ (orange line).

$\sim 6 x$ at $T=100 \mathrm{~K}$ in Fig. 4(a)]. This possibly explains the exciting experimental report of magnetic field induced $300 \%$ enhancement of the electronic component of the longitudinal thermal conductivity in the WSM phase of $\mathrm{Bi}_{89} \mathrm{Sb}_{11}$ [43]. We also find a similar enhancement in $S_{x y}$ and $\kappa_{x y}$, as shown in Fig. 4(b), and also in $\alpha$.

\section{VIOLATION OF WIEDEMANN-FRANZ LAW AND MOTT RELATION}

From a theoretical perspective, the violation of the Wiedemann-Franz law and the Mott relations (solely for the electronic transport coefficients) arises from the breakdown of the conditions necessary for the Sommerfeld expansion [38]. This simply manifests as the variation of the Lorentz number (or the Mott number) from its ideal value of $\mathcal{L}_{0}=$ $\pi^{2} k_{B}^{2} /\left(3 e^{2}\right)$ [or $\mathcal{M}_{0}=\pi^{2} k_{B}^{2} /(3 e)$ ] [38]. Such a violation of the Wiedemann-Franz law can also arise in other nontopological semimetals owing to the comparable values of $\mu$ and $T$.

However, in addition to this the anomaly induced transport in WSMs causes the violation of the Wiedemann-Franz law and the Mott relations, which goes much beyond the breakdown of the Sommerfeld expansion. This is shown explicitly in Figs. 4(c) and 4(d) where we plot the ratio $L(B, T) / L_{0}(T)$ and $M(B, T) / M_{0}(T)$, where $L_{0}$ and $M_{0}$ are the corresponding Drude counterpart. The corresponding temperature dependence of the Drude Lorentz number $\left(L_{0}\right)$ and Mott number $\left(M_{0}\right)$ in WSMs is shown in Fig. 5.

The earlier reports of violation of the Wiedemann-Franz law in WSMs $[25,29,33]$ have predominantly focused on the intranode scattering contribution. In contrast, the violation discussed above is essentially a chiral anomaly induced phenomena.

\section{DISCUSSION}

Our derivation of transport coefficients is valid in the semiclassical regime, where the Landau quantization can be ignored. This happens for $\omega_{c} \tau \ll 1$ where $\omega_{c}=v_{F} / l_{B}$ is the cyclotron frequency with magnetic length $l_{B}=\sqrt{\hbar /(e B)}$. In experiments, the semiclassical magneto-resistance regime with $\delta \sigma \propto B^{2}$ has been observed for magnetic field values of up to $6 \mathrm{~T}$, depending on the material $[16,18,27,44]$.

The use of the Boltzmann kinetic equation is valid in the weak scattering limit where $\mu \gg \hbar / \tau$. Furthermore, the formulation used here assumes that each of Weyl nodes has a distinct Fermi surface. This works well in the regime where the chemical potential is smaller than half of the band inversion gap from which the Weyl nodes originate [45].

Note that other mechanisms have also been reported to give rise to anomalous transport. For example, inhomogeneous current distribution in samples (current jetting) has been shown to give rise to negative MR posing questions about the "intrinsic" nature of the observed quantities [8,44,46]. Negative MR can also arise in nontopological materials owing to microscopic disorder, as shown in GaAs quantum wells [47]. From a theoretical perspective, even in the absence of chiral anomalies, the Berry curvature can give rise to negative MR [48].

\section{CONCLUSION}

To summarize, we have predicted an additional quantum anomaly in chiral fluids, the thermal chiral anomaly, which pumps chiral charge from one node to other in the presence of $\nabla T \cdot \mathbf{B} \neq 0$. We have shown that in addition to planar Hall and planar Nernst effects, the planar Ettinghausen and Righi-Leduc effects also arise from these anomalies. We predict a significant enhancement in the magnetic field induced thermoelectric conductivity, Seebeck effect, Nernst effect, and thermal conductivity with increasing temperature. We also demonstrate that the chiral anomaly induced magnetotransport in WSMs violates the Wiedemann-Franz law and Mott relation at finite temperatures. Our work provides a robust framework for exploring and interpreting the impact of chiral anomalies in magneto-transport experiments in WSMs.

\section{ACKNOWLEDGMENTS}

A.A. acknowledges the Science Education and Research Board (SERB) and the Department of Science and Technology (DST) of the government of India for financial support.

\section{APPENDIX A: COEFFICIENT OF ANOMALIES}

The coefficients of the three chiral anomalies are explicitly given by the following expression:

$$
\mathcal{C}_{n}^{s}=\frac{1}{n !} \int \frac{d \mathbf{k}}{8 \pi^{3}}\left(\frac{e}{\hbar} \mathbf{v}^{s} \cdot \boldsymbol{\Omega}^{s}\right)\left(\frac{\epsilon^{s}-\mu}{T}\right)^{n}\left(\frac{-\partial f^{s}}{\partial \epsilon_{s}}\right) .
$$

Considering an isotropic Weyl cone with linearly dispersing nodes, the coefficients of anomalies can be calculated to be

$$
\left\{\mathcal{C}_{0}^{s}, \mathcal{C}_{1}^{s}, \mathcal{C}_{2}^{s}\right\}=-s \frac{e}{4 \pi^{2} \hbar^{2}}\left\{\mathcal{F}_{0}(\beta \mu), k_{B} \mathcal{F}_{1}(\beta \mu), \frac{k_{B}^{2}}{2} \mathcal{F}_{2}(\beta \mu)\right\} .
$$

In the limit $\beta \mu \rightarrow \infty$ we have $\mathcal{C}_{0}^{s}=-\frac{s}{4 \pi^{2} \hbar^{2}}, \mathcal{C}_{1}^{s}=0$, and $\mathcal{C}_{2}^{s}=-\frac{s k_{B}^{2}}{24 \hbar^{2}}$. The temperature dependence of these functions is shown in Fig. 6. Note that the zero-temperature limit of $\mathcal{C}_{2}$ is identical to the coefficient of the relativistic chiral gravitational anomaly $[2,4,5]$. This is why the thermal transport in 


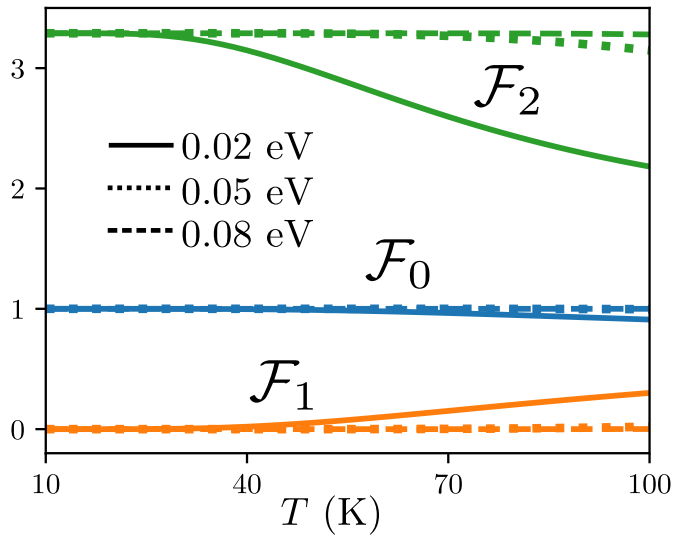

FIG. 6. The temperature dependence of the dimensionless part of the coefficients of the quantum chiral anomalies: $\mathcal{F}_{0}, \mathcal{F}_{1}$, and $\mathcal{F}_{2}$. Each of these functions are plotted with temperature for three different values of chemical potentials: $\mu=0.02 \mathrm{eV}$ (solid lines), $\mu=0.05 \mathrm{eV}$ (dotted lines), and $\mu=0.08 \mathrm{eV}$ (dashed lines). Note that $\mathcal{F}_{1} \rightarrow 0$ in the low-temperature limit.

WSMs is considered to be an analog of the relativistic chiral gravitational anomaly even in flat space-time [5].

We emphasize that in Eqs. (1) and (2), the $\mathcal{C}_{1}^{s}$ term was missed in earlier studies, as it vanishes in the $\beta \mu \rightarrow \infty$ limit. Our study captures it in the general equation for the equilibrium current, and more specific expressions [Eq. (A2)] in the finite but large $\beta \mu$ calculations [31]. Since this term is related to chiral charge pumping in the presence of a temperature gradient, we refer to it as the thermal chiral anomaly.

\section{APPENDIX B: CHIRAL CHEMICAL POTENTIAL AND TEMPERATURE}

For $\delta \mu^{s} \ll \mu$ and $\delta T^{s} \ll T$, we can rewrite the collision integral for node $s$ as

$$
\begin{aligned}
I_{\text {coll }}^{s}= & -\frac{\delta g^{s}}{\tau^{*}}+\frac{1}{\tau_{v}}\left[\delta \mu^{s}-\delta \mu^{\bar{s}}+\left(\delta T^{s}-\delta T^{\bar{s}}\right) \frac{\tilde{\epsilon}^{s}-\mu}{T}\right] \\
& \times\left(\partial_{\tilde{\epsilon}} \tilde{f}^{s}\right) .
\end{aligned}
$$

Here, we have defined $\frac{1}{\tau^{*}}=\frac{1}{\tau_{0}}+\frac{1}{\tau_{v}}$ and $\delta g^{s}=g^{s}-$ $f\left(\tilde{\epsilon}^{s}, \mu^{s}, T^{s}\right)$. Using Eq. (B1), the Boltzmann kinetic equation can be written as

$$
\begin{aligned}
& \Delta^{s}\left(\tilde{\mathbf{v}}^{s}+\frac{e}{\hbar}\left(\tilde{\mathbf{v}}^{s} \cdot \boldsymbol{\Omega}^{s}\right) \mathbf{B}\right) \cdot\left(e \mathbf{E}+\frac{\tilde{\epsilon}^{s}-\mu}{T} \nabla T\right) \partial_{\tilde{\epsilon}} \tilde{f}^{s} \\
& \quad=\frac{\delta g^{s}}{\tau^{*}}-\frac{1}{\tau_{v}}\left[\delta \mu^{s}-\delta \mu^{\bar{s}}+\left(\delta T^{s}-\delta T^{\bar{s}}\right) \frac{\tilde{\epsilon}^{s}-\mu}{T}\right] \partial_{\tilde{\epsilon}} \tilde{f}^{s} .
\end{aligned}
$$

In order to find the chiral chemical potential and chiral temperature, we construct the following equations. Using $\delta \mu^{s}=$ $-\delta \mu^{\bar{s}}$ and $\delta T^{s}=-\delta T^{\bar{s}}$, which is true for identical density of states of both cones, and integrating Eq. (B2), we get

$$
\delta \mu^{s} \mathcal{D}_{0}^{s}+\delta T^{s} \frac{1}{T} \mathcal{D}_{1}^{s}=-\frac{\tau_{v}}{2}\left(e \mathbf{E} \cdot \boldsymbol{\Lambda}_{0}^{s}+\frac{1}{T} \nabla T \cdot \boldsymbol{\Lambda}_{1}^{s}\right) .
$$

Multiplying Eq. (B2) with $\left(\tilde{\epsilon}_{s}-\mu\right)$ and integrating we obtain

$$
\delta \mu^{s} \mathcal{D}_{1}^{s}+\delta T^{s} \frac{1}{T} \mathcal{D}_{2}^{s}=-\frac{\tau_{v}}{2}\left(e \mathbf{E} \cdot \boldsymbol{\Lambda}_{1}^{s}+\frac{1}{T} \nabla T \cdot \boldsymbol{\Lambda}_{2}^{s}\right) .
$$

We find that the equilibrium band velocity and orbital magnetization do not contribute to $\boldsymbol{\Lambda}_{n}^{s}$ at all. It gets a contribution only from the chiral magnetic velocity and thus we have $\boldsymbol{\Lambda}_{n}^{s}=\Lambda_{n}^{s} \mathbf{B}$ with $\Lambda_{n}^{s} \propto s$. Here, we emphasize that for going beyond the constant $\tau_{v}$ approximation, we have to include the energy and temperature dependence of $\tau_{v}$ in the above equations. In the linear-response regime, all the energy integrals for transport coefficients contain the derivative of the Fermi function. Consequently, the Fermi-surface averaged value of the scattering time with its energy (chemical potential) and temperature dependence has to be substituted in Eq. (B3), i.e., $\tau_{v} \rightarrow \tau_{v}(\mu, T)$. Details of the energy and temperature dependence of the scattering time is discussed further in Appendix D.

Solving Eqs. (B3) and (B4), we obtain the chiral chemical potential and chiral temperature:

$$
\begin{aligned}
\delta \mu^{s}= & -\frac{\tau_{v}}{2} \frac{\mathcal{D}_{1}^{s} \mathcal{D}_{2}^{s}}{\operatorname{det}\left[\mathcal{D}^{s}\right]}\left[\left(\frac{\boldsymbol{\Lambda}_{0}^{s}}{\mathcal{D}_{1}^{s}}-\frac{\boldsymbol{\Lambda}_{1}^{s}}{\mathcal{D}_{2}^{s}}\right) \cdot e \mathbf{E}\right. \\
& \left.+\left(\frac{\boldsymbol{\Lambda}_{1}^{s}}{\mathcal{D}_{1}^{s}}-\frac{\boldsymbol{\Lambda}_{2}^{s}}{\mathcal{D}_{2}^{s}}\right) \cdot \frac{\nabla T}{T}\right], \\
\frac{\delta T^{s}}{T}= & -\frac{\tau_{v}}{2} \frac{\mathcal{D}_{0}^{s} \mathcal{D}_{1}^{s}}{\operatorname{det}\left[\mathcal{D}^{s}\right]}\left[\left(\frac{\boldsymbol{\Lambda}_{1}^{s}}{\mathcal{D}_{1}^{s}}-\frac{\boldsymbol{\Lambda}_{0}^{s}}{\mathcal{D}_{0}^{s}}\right) \cdot e \mathbf{E}\right. \\
& \left.+\left(\frac{\boldsymbol{\Lambda}_{2}^{s}}{\mathcal{D}_{1}^{s}}-\frac{\boldsymbol{\Lambda}_{1}^{s}}{\mathcal{D}_{0}^{s}}\right) \cdot \frac{\nabla T}{T}\right] .
\end{aligned}
$$

Here, $\operatorname{det}\left[\mathcal{D}^{s}\right] \equiv \mathcal{D}_{0}^{s} \mathcal{D}_{2}^{s}-\left(\mathcal{D}_{1}^{s}\right)^{2}$. This highlights the fact that in the presence of either a $\mathbf{E} \cdot \mathbf{B} \neq 0$ term or a $\nabla T \cdot \mathbf{B} \neq 0$ term, the Weyl nodes develop a finite $\delta \mu^{s}$ as well as $\delta T^{s}$.

\section{APPENDIX C: ANOMALY INDUCED CURRENTS AND TRANSPORT COEFFICIENTS}

Solving the Boltzmann kinetic equation (B2), we obtain the nonequilibrium distribution function to be

$$
\begin{aligned}
g^{s}= & f\left(\tilde{\epsilon}^{s}, \mu^{s}, T^{s}\right)-\tau^{*}\left[\frac{2}{\tau_{v}}\left(\delta \mu^{s}+\delta T^{s} \frac{\tilde{\epsilon}^{s}-\mu}{T}\right)\right. \\
& \left.+\Delta^{s} \mathbf{v}_{\mathbf{B}}^{s}\left(e \mathbf{E}+\left(\tilde{\epsilon}^{s}-\mu\right) \frac{\nabla T}{T}\right)\right]\left(-\frac{\partial \tilde{f}^{s}}{\partial \tilde{\epsilon}^{s}}\right) .
\end{aligned}
$$

Here, we have defined $\mathbf{v}_{\mathbf{B}}^{s}=\tilde{\mathbf{v}}^{s}+\frac{e}{\hbar}\left(\tilde{\mathbf{v}}^{s} \cdot \boldsymbol{\Omega}^{s}\right) \mathbf{B}$. Note that in the chiral limit, $\tau_{v} \gg \tau^{*}$, the dominant contribution comes from the first term of the distribution function. This condition is viable due to the large momentum transfer required for internode scattering. Using this distribution function and the expressions of chiral chemical potential and temperature in the chiral limit we have

$$
\begin{aligned}
\mathbf{j}_{e}^{s}= & \frac{e \tau_{v}}{2}\left[\mathbf{\Lambda}_{0}^{s} \frac{\mathcal{D}_{1}^{s} \mathcal{D}_{2}^{s}}{\operatorname{det}\left[\mathcal{D}^{s}\right]}\left(\mathbf{L}_{12}^{01} \cdot e \mathbf{E}+\mathbf{L}_{12}^{12} \cdot \frac{\nabla T}{T}\right)\right. \\
& \left.+\boldsymbol{\Lambda}_{1}^{s} \frac{\mathcal{D}_{0}^{s} \mathcal{D}_{1}^{s}}{\operatorname{det}\left[\mathcal{D}^{s}\right]}\left(\mathbf{L}_{10}^{10} \cdot e \mathbf{E}+\mathbf{L}_{10}^{21} \cdot \frac{\nabla T}{T}\right)\right] .
\end{aligned}
$$




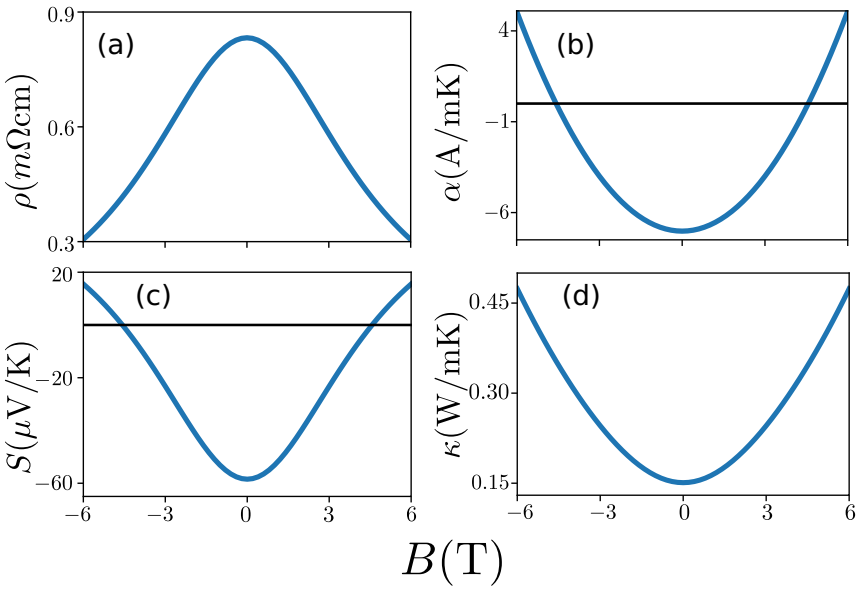

FIG. 7. Variation of longitudinal transport coefficients as a function of $B$ : (a) resistivity, (b) thermoelectric coefficient, (c) Seebeck coefficient, and (d) thermal conductivity. We have used the same parameters here as in Fig. 3.

Here, we have defined $\mathbf{L}_{l m}^{i j}=\frac{\boldsymbol{\Lambda}_{i}^{s}}{\mathcal{D}_{l}^{s}}-\frac{\boldsymbol{\Lambda}_{j}^{s}}{\mathcal{D}_{l m}^{s}}$. From this expression one can immediately have an idea of the dominant contributions to the charge current. For instance, since $\boldsymbol{\Lambda}_{1}^{s} \rightarrow$ 0 in the limit $\beta \mu \rightarrow \infty$, in this limit the second contribution in Eq. (C2) goes to zero. Similar to the charge current, the heat current can be written as

$$
\begin{aligned}
\mathbf{j}_{Q}^{s}= & -\frac{\tau_{v}}{2}\left[\mathbf{\Lambda}_{1}^{s} \frac{\mathcal{D}_{1}^{s} \mathcal{D}_{2}^{s}}{\operatorname{det}\left[\mathcal{D}^{s}\right]}\left(\mathbf{L}_{12}^{01} \cdot e \mathbf{E}+\mathbf{L}_{12}^{12} \cdot \frac{\nabla T}{T}\right)\right. \\
& \left.+\mathbf{\Lambda}_{2}^{s} \frac{\mathcal{D}_{0}^{s} \mathcal{D}_{1}^{s}}{\operatorname{det}\left[\mathcal{D}^{s}\right]}\left(\mathbf{L}_{10}^{10} \cdot e \mathbf{E}+\mathbf{L}_{10}^{21} \cdot \frac{\nabla T}{T}\right)\right] .
\end{aligned}
$$

We find that for a reasonable set of experimental parameters, the dominant contribution in the heat current comes from the second term.

Reading off the transport coefficients from Eqs. (C2) and (C3), we have $\delta \sigma_{i j}^{s} \propto \mathcal{D}_{0}{ }^{s} \Lambda_{1 i}^{s} \Lambda_{1 j}^{s}+\mathcal{D}_{2}{ }^{s} \Lambda_{0 i}^{s} \Lambda_{0 j}^{s}-$ $2 \mathcal{D}_{2}{ }^{s} \Lambda_{0 i}^{s} \Lambda_{1 j}^{s}$. Clearly, the $B$ induced correction to the charge conductivity comprises the ECA $\left(\Lambda_{0 i}^{s} \Lambda_{0 j}^{s}\right)$, TCA $\left(\Lambda_{1 i}^{s} \Lambda_{1 j}^{s}\right)$, and mixed ECA-TCA $\left(\Lambda_{0 i}^{s} \Lambda_{1 j}^{s}\right)$ anomaly. The anomaly induced thermoelectric conductivity $\delta \alpha_{i j}^{s} \propto \Lambda_{1 i}^{s}\left(\mathcal{D}_{1}{ }^{s} \Lambda_{1 j}^{s}-\mathcal{D}_{0}{ }^{s} \Lambda_{2 j}^{s}-\mathcal{D}_{2}{ }^{s} \Lambda_{0 j}^{s}\right)+\mathcal{D}_{1}{ }^{s} \Lambda_{0 i}^{s} \Lambda_{2 j}^{s}$. It arises from TCA, mixed ECA-TCA, mixed GCA $\left(\Lambda_{0 i}^{s} \Lambda_{2 j}^{s}\right)$, and mixed GCA-TCA $\left(\Lambda_{1 i}^{s} \Lambda_{2 j}^{s}\right)$ anomaly. Similarly we have $\delta \bar{\kappa}_{i j}^{s} \propto \mathcal{D}_{0}{ }^{s} \Lambda_{2 i}^{s} \Lambda_{2 j}^{s}+\mathcal{D}_{2}{ }^{s} \Lambda_{1 i}^{s} \Lambda_{1 j}^{s}-2 \mathcal{D}_{1}{ }^{s} \Lambda_{2 i}^{s} \Lambda_{1 j}^{s}$. It has signatures of TCA, GCA, and mixed TCA-GCA anomaly $\left(\Lambda_{1 i}^{s} \Lambda_{2 j}^{s}\right)$.

In Fig. 7 we show the longitudinal transport coefficients as a function of the applied magnetic field. Panel (a) shows the resistivity, and the obtained value is consistent with the measured resistivity in $\mathrm{Na}_{3} \mathrm{Bi}$ [16]. Panels (b) and (d) show the thermoelectric conductivity and Seebeck coefficient, whose values are broadly consistent with the experiment values $[26,27]$. In panel (d) we have plotted the thermal conductivity which is also consistent with experiment [43].

\section{APPENDIX D: ENERGY AND TEMPERATURE DEPENDENCE OF THE SCATTERING TIME}

As discussed earlier, the energy and temperature dependence of the scattering coefficients can be included in our formalism via the substitution $\tau_{0} \rightarrow \tau_{0}(\mu, T)$ and $\tau_{v} \rightarrow$ $\tau_{v}(\mu, T)$. The energy and temperature dependence of the intranode scattering time has been discussed extensively in Ref. [49]. For the short-range disorder modelled as neutral point defects, the scattering time has been calculated [49] to be $\tau_{\delta} \propto 1 / \mu^{2}\left(1-e^{-T / T_{F}}\right)$, with $T_{F}$ being the Fermi temperature. For the charged impurities, within Thomas-Fermi screening approximation, the scattering time is given by [49] $\tau_{c} \propto \mu^{2}\left[1+2 \pi^{2} T^{2} /\left(3 T_{F}^{2}\right)\right]$. Furthermore, the electronphonon scattering time due to the acoustic phonon, in the degenerate or Bloch-Gruneisen regime, has been shown [49] to be $1 / \tau_{p} \propto\left(k_{B} T\right)^{5} / \mu^{2}$. In contrast, the detailed analysis of the internode scattering and its energy and temperature dependence is still an open problem. A simple estimation of $\tau_{v}$ assuming screened charged impurities [40] yields $\tau_{v} \sim$ $\mu^{2} V(2 \mathbf{b})$, where $\mathbf{b}$ denotes the internode separation, and $V$ is the fourier transform of the screened potential.
[1] H. B. Nielsen and M. Ninomiya, The Adler-Bell-Jackiw anomaly and Weyl fermions in a crystal, Phys. Lett. B 130, 389 (1983).

[2] K. Landsteiner, E. Megías, and F. Pena-Benitez, Gravitational Anomaly and Transport Phenomena, Phys. Rev. Lett. 107, 021601 (2011).

[3] D. T. Son and B. Z. Spivak, Chiral anomaly and classical negative magnetoresistance of Weyl metals, Phys. Rev. B 88, 104412 (2013).

[4] A. Lucas, R. A. Davison, and S. Sachdev, Hydrodynamic theory of thermoelectric transport and negative magnetoresistance in Weyl semimetals, Proc. Natl. Acad. Sci. USA 113, 9463 (2016).

[5] J. Gooth, A. C. Niemann, T. Meng, A. G. Grushin, K. Landsteiner, B. Gotsmann, F. Menges, M. Schmidt, C. Shekhar, V. Süß, R. Hühne, B. Rellinghaus, C. Felser, B. Yan, and K. Nielsch, Experimental signatures of the mixed axial-gravitational anomaly in the Weyl semimetal nbp, Nature (London) 547, 324 (2017).

[6] M. N. Chernodub, A. Cortijo, and M. A. H. Vozmediano, Generation of a Nernst Current from the Conformal Anomaly in Dirac and Weyl Semimetals, Phys. Rev. Lett. 120, 206601 (2018).

[7] X. Wan, A. M. Turner, A. Vishwanath, and S. Y. Savrasov, Topological semimetal and fermi-arc surface states in the electronic structure of pyrochlore iridates, Phys. Rev. B 83, 205101 (2011).

[8] N. P. Armitage, E. J. Mele, and A. Vishwanath, Weyl and Dirac semimetals in three-dimensional solids, Rev. Mod. Phys. 90, 015001 (2018).

[9] S.-Y. Xu, I. Belopolski, N. Alidoust, M. Neupane, G. Bian, C. Zhang, R. Sankar, G. Chang, Z. Yuan, C.-C. Lee, S.-M. Huang, H. Zheng, J. Ma, D. S. Sanchez, B. Wang, A. Bansil, F. Chou, P. P. Shibayev, H. Lin, S. Jia, and M. Z. Hasan, Discovery of 
a Weyl fermion semimetal and topological fermi arcs, Science 349, 613 (2015).

[10] S.-M. Huang, S.-Y. Xu, I. Belopolski, C.-C. Lee, G. Chang, B. Wang, N. Alidoust, G. Bian, M. Neupane, C. Zhang, S. Jia, A. Bansil, H. Lin, and M. Z. Hasan, A Weyl fermion semimetal with surface fermi arcs in the transition metal monopnictide taas class, Nat. Commun. 6, 7373 (2015).

[11] A. A. Soluyanov, D. Gresch, Z. Wang, Q. Wu, M. Troyer, X. Dai, and B. A. Bernevig, Type-ii Weyl semimetals, Nature (London) 527, 495 (2015).

[12] M. Z. Hasan, S.-Y. Xu, I. Belopolski, and S.-M. Huang, Discovery of Weyl fermion semimetals and topological fermi arc states, Annu. Rev. Condens. Matter Phys. 8, 289 (2017).

[13] D. T. Son and N. Yamamoto, Berry Curvature, Triangle Anomalies, and the Chiral Magnetic Effect in Fermi Liquids, Phys. Rev. Lett. 109, 181602 (2012).

[14] A. A. Zyuzin and A. A. Burkov, Topological response in Weyl semimetals and the chiral anomaly, Phys. Rev. B 86, 115133 (2012).

[15] S.-K. Yip, Kinetic equation and magneto-conductance for Weyl metal in the clean limit, arXiv:1508.01010.

[16] J. Xiong, S. K. Kushwaha, T. Liang, J. W. Krizan, M. Hirschberger, W. Wang, R. J. Cava, and N. P. Ong, Evidence for the chiral anomaly in the Dirac semimetal $\mathrm{Na}_{3} \mathrm{Bi}$, Science 350, 413 (2015).

[17] X. Huang, L. Zhao, Y. Long, P. Wang, D. Chen, Z. Yang, H. Liang, M. Xue, H. Weng, Z. Fang, X. Dai, and G. Chen, Observation of the Chiral-Anomaly-Induced Negative Magnetoresistance in 3d Weyl Semimetal Taas, Phys. Rev. X 5, 031023 (2015).

[18] H. Li, H. He, H.-Z. Lu, H. Zhang, H. Liu, R. Ma, Z. Fan, S.-Q. Shen, and J. Wang, Negative magnetoresistance in Dirac semimetal $\mathrm{Cd}_{3} \mathrm{As} 2$, Nat. Commun. 7, 10301 (2016).

[19] Q. Li, D. E. Kharzeev, C. Zhang, Y. Huang, I. Pletikosić, A. V. Fedorov, R. D. Zhong, J. A. Schneeloch, G. D. Gu, and T. Valla, Chiral magnetic effect in $\mathrm{ZrTe}_{5}$, Nat. Phys. 12, 550 (2016).

[20] A. A. Burkov, Giant planar hall effect in topological metals, Phys. Rev. B 96, 041110(R) (2017).

[21] S. Nandy, G. Sharma, A. Taraphder, and S. Tewari, Chiral Anomaly as the Origin of the Planar Hall Effect in Weyl Semimetals, Phys. Rev. Lett. 119, 176804 (2017).

[22] A. Thakur, K. Sadhukhan, and A. Agarwal, Dynamic currentcurrent susceptibility in three-dimensional Dirac and Weyl semimetals, Phys. Rev. B 97, 035403 (2018).

[23] K. Sonowal, A. Singh, and A. Agarwal, Giant optical activity and kerr effect in type-i and type-ii Weyl semimetals, Phys. Rev. B 100, 085436 (2019).

[24] R. Lundgren, P. Laurell, and G. A. Fiete, Thermoelectric properties of Weyl and Dirac semimetals, Phys. Rev. B 90, 165115 (2014).

[25] K.-S. Kim, Role of axion electrodynamics in a Weyl metal: Violation of Wiedemann-Franz law, Phys. Rev. B 90, 121108(R) (2014).

[26] M. Hirschberger, S. Kushwaha, Z. Wang, Q. Gibson, S. Liang, C. A. Belvin, B. A. Bernevig, R. J. Cava, and N. P. Ong, The chiral anomaly and thermopower of Weyl fermions in the halfheusler gdptbi, Nat. Mater. 15, 1161 (2016).
[27] Z. Jia, C. Li, X. Li, J. Shi, Z. Liao, D. Yu, and X. Wu, Thermoelectric signature of the chiral anomaly in $\mathrm{Cd}_{3} \mathrm{As}_{2}$, Nat. Commun. 7, 13013 (2016).

[28] B. Z. Spivak and A. V. Andreev, Magnetotransport phenomena related to the chiral anomaly in Weyl semimetals, Phys. Rev. B 93, 085107 (2016).

[29] G. Sharma, P. Goswami, and S. Tewari, Nernst and magnetothermal conductivity in a lattice model of Weyl fermions, Phys. Rev. B 93, 035116 (2016).

[30] U. Stockert, R. D. dos Reis, M. O. Ajeesh, S. J. Watzman, M. Schmidt, C. Shekhar, J. P. Heremans, C. Felser, M. Baenitz, and M. Nicklas, Thermopower and thermal conductivity in the Weyl semimetal NbP, J. Phys.: Condens. Matter 29, 325701 (2017).

[31] V. A. Zyuzin, Magnetotransport of Weyl semimetals due to the chiral anomaly, Phys. Rev. B 95, 245128 (2017).

[32] G. Sharma and S. Tewari, Transverse thermopower in Dirac and Weyl semimetals, Phys. Rev. B 100, 195113 (2019).

[33] S. Nandy, A. Taraphder, and S. Tewari, Planar thermal Hall effect in Weyl semimetals, Phys. Rev. B 100, 115139 (2019).

[34] G. Sundaram and Q. Niu, Wave-packet dynamics in slowly perturbed crystals: Gradient corrections and berry-phase effects, Phys. Rev. B 59, 14915 (1999).

[35] D. Xiao, M.-C. Chang, and Q. Niu, Berry phase effects on electronic properties, Rev. Mod. Phys. 82, 1959 (2010).

[36] D. Xiao, J. Shi, and Q. Niu, Berry Phase Correction to Electron Density of States in Solids, Phys. Rev. Lett. 95, 137204 (2005).

[37] D. Xiao, Y. Yao, Z. Fang, and Q. Niu, Berry-Phase Effect in Anomalous Thermoelectric Transport, Phys. Rev. Lett. 97, 026603 (2006).

[38] N. W. Ashcroft and N. D. Mermin, Solid State Physics, HRW International Editions (Holt, Rinehart and Winston, New York, 1976).

[39] K. Das and A. Agarwal, Linear magnetochiral transport in tilted type-i and type-ii Weyl semimetals, Phys. Rev. B 99, 085405 (2019).

[40] S. A. Parameswaran, T. Grover, D. A. Abanin, D. A. Pesin, and A. Vishwanath, Probing the Chiral Anomaly with Nonlocal Transport in Three-Dimensional Topological Semimetals, Phys. Rev. X 4, 031035 (2014).

[41] K. Das and A. Agarwal, Berry curvature induced thermopower in type-i and type-ii Weyl semimetals, Phys. Rev. B 100, 085406 (2019).

[42] C. Schindler, S. Galeski, S. N. Guin, W. Schnelle, N. Kumar, C. Fu, H. Borrmann, C. Shekhar, Y. Zhang, Y. Sun, C. Felser, T. Meng, A. G. Grushin, and J. Gooth, Observation of an anomalous heat current in a Weyl fermion semimetal, arXiv: 1810.02300 .

[43] D. Vu, W. Zhang, C. Şahin, M. Flatté, N. Trivedi, and J. P. Heremans, Thermal chiral anomaly in the magnetic-field induced ideal Weyl phase of Bi89Sb11, arXiv:1906.02248.

[44] S. Liang, J. Lin, S. Kushwaha, J. Xing, N. Ni, R. J. Cava, and N. P. Ong, Experimental Tests of the Chiral Anomaly Magnetoresistance in the Dirac-Weyl Semimetals $\mathrm{Na}_{3} \mathrm{Bi}$ and GdPtBi, Phys. Rev. X 8, 031002 (2018).

[45] H. Ishizuka and N. Nagaosa, Robustness of anomaly-related magnetoresistance in doped Weyl semimetals, Phys. Rev. B 99, 115205 (2019).

[46] F. Arnold, C. Shekhar, S.-C. Wu, Y. Sun, R. D. dos Reis, N. Kumar, M. Naumann, M. O. Ajeesh, M. Schmidt, 
A. G. Grushin, J. H. Bardarson, M. Baenitz, D. Sokolov, H. Borrmann, M. Nicklas, C. Felser, E. Hassinger, and B. Yan, Negative magnetoresistance without well-defined chirality in the Weyl semimetal tap, Nat. Commun. 7, 11615 (2016).

[47] J. Xu, M. K. Ma, M. Sultanov, Z.-L. Xiao, Y.-L. Wang, D. Jin, Y.-Y. Lyu, W. Zhang, L. N. Pfeiffer, K. W. West, K. W. Baldwin, M. Shayegan, and W.-K. Kwok, Negative longitudinal magnetoresistance in gallium arsenide quantum wells, Nat. Commun. 10, 287 (2019).

[48] Y. Gao, S. A. Yang, and Q. Niu, Intrinsic relative magnetoconductivity of nonmagnetic metals, Phys. Rev. B 95, 165135 (2017).

[49] S. Das Sarma, E. H. Hwang, and H. Min, Carrier screening, transport, and relaxation in three-dimensional Dirac semimetals, Phys. Rev. B 91, 035201 (2015). 